\title{
FILOSOFÍA AMBIENTAL DE CAMPO: EDUCACIÓN E INVESTIGACIÓN PARA LA VALORACIÓN ECOLÓGICA Y ÉTICA DE LOS INSECTOS DULCEACUÍCOLAS ${ }^{1}$
}

\author{
JAVIER RENDOLL CÁRCAMO ${ }^{\mathrm{a}, \mathrm{b}, \mathrm{c}}$, TAMARA CONTADOR ${ }^{\mathrm{a}, \mathrm{b}, \mathrm{d}}$, MELISA GAÑÁN ${ }^{\mathrm{a}}$, \\ NANCYROSE HOUSTON ${ }^{\mathrm{b}}$, MIGUEL TRONCOSO ${ }^{\mathrm{b}}$, GONZALO ARRIAGADA $^{\mathrm{a}}$, \\ CAMILA SALDÍAS ${ }^{b}$, PAULA CABALLERO ${ }^{e}$, JAVIERA MALEBRÁN $^{b}$, \\ JAMES KENNEDYa,f, PETER CONVEY \& RICARDO ROZZI ${ }^{\mathrm{b}, \mathrm{h}}$

\section{RESUMEN}

En un mundo aceleradamente cambiante, para confrontar las pérdidas de biodiversidad, el desconocimiento y falta de valoración de los grupos de organismos más diversos, son necesarios cambios culturales que trasciendan a los ámbitos puramente científicos y tecnológicos. En este trabajo se aborda esta problemática al enfocarse en uno de los grupos de organismos menos conocidos en una de las regiones más remotas del planeta: los insectos dulceacuícolas en la ecorregión subantártica de Magallanes. El desarrollo de esta tesis consideró investigaciones científico-ecológicas y filosófico-ambientales que se integraron con prácticas de educación ambiental formal y no-formal que valoran a los insectos dulceacuícolas, particularmente como indicadores del cambio climático. La integración de las ciencias y la filosofía se realizó adaptando metodología de la Filosofía Ambiental de Campo que incluye un ciclo de cuatro pasos. La investigación transdisciplinaria sobre los insectos dulceacuícolas y sus ecosistemas subantárticos fue la base para la composición de metáforas y actividades educativas con escolares, otros miembros de la comunidad local y visitantes al Parque Omora, en Puerto Williams, Chile. A partir de esta tesis se diseñaron nuevas actividades educativas al aire libre cuyos objetivos son despertar el interés de la ciudadanía por los insectos, orientar las percepciones sobre estos organismos, sus hábitats y hábitos de vida. De esta manera, este trabajo aspira, a nivel local, a contribuir a un mayor conocimiento, valoración y conservación de esta singular biodiversidad subantártica y, a nivel global, a superar la subvaloración del grupo más diverso de organismos: los insectos.

a Laboratorio Wankara de Ecosistemas Dulceacuícolas Antárticos y Subantárticos, Universidad de Magallanes, Chile.

$\bigotimes$ javier.rendoll@gmail.com

b Programa de Conservación Biocultural Subantártica, Instituto de Ecología y Biodiversidad, Chile

c Programa de Doctorado en Ciencias Antárticas y Subantárticas, Universidad de Magallanes, Chile

Núcleo Milenio de Salmónidos Invasores, INVASAL, Chile

Programa de Difusión de Ciencias, Instituto de Ecología y Biodiversidad, Chile

Department of Biology, University of North Texas, USA

British Antarctic Survey, UK

h Department of Philosophy and Religion, University of North Texas, USA

1 Este artículo se basa en el trabajo de tesis de Magíster en Ciencias de la Universidad de Magallanes (Programa de Magíster en Ciencias, mención Manejo y Conservación de Recursos Naturales en Ambientes Subantárticos), realizado por Javier Rendoll Cárcamo en colaboración con un conjunto de investigadores y educadores que practican la FILAC. 
PALABRAS CLAVE: cambio climático, conservación, estudios socio-ecológicos a largo-plazo, invertebrados, Magallanes, subantártico.

\title{
FIELD ENVIRONMENTAL PHILOSOPHY: EDUCATION AND RESEARCH FOR THE ECOLOGICAL AND ETHICAL APPRECIATION OF FRESHWATER INSECTS
}

\begin{abstract}
In a rapidly changing world, to confront biodiversity losses and the lack of appreciation for and knowledge about the most diverse groups of organisms, it is urgently necessary to stimulate cultural shifts that transcend purely scientific and technological domains. This paper addresses this problem by focusing on one of the least known groups of organisms, and in one of the most remote regions of the planet: freshwater insects in the sub-Antarctic Magellanic ecoregion. The work of this thesis included scientific-ecological and environmental philosophical research that was integrated into formal and non-formal environmental education practices that value freshwater insects, particularly as indicators of climate change. The integration of science and philosophy was done adapting the Field Environmental Philosophy methodology that includes a four-step cycle. Transdisciplinary research on freshwater insects and their sub-Antarctic ecosystems served as a basis for the composition of metaphors and educational activities with schoolchildren, other members of the local community and visitors to Omora Park, in Puerto Williams, Chile. Based on this work, new outdoor educational activities were designed with the objective of awakening the interest of citizens for insects, and nurturing their perceptions about these organisms, their habitats and life habits. In this way, at a local scale this work aims to contribute to greater knowledge, appreciation and conservation of this unique sub-Antarctic biodiversity, and at a global scale it aims to contribute overcoming the under-appreciation for the most diverse group of organisms: the insects.
\end{abstract}

KEY WORDS: climate change, conservation, long-term socio-ecological research, invertebrates, Magallanes, subantarctic.

\section{INTRODUCCIÓN}

La ecorregión subantártica de Magallanes incluye los bosques y humedales más australes del planeta (Rozzi et al. 2012) que por su condición remota han experimentado escaso impacto antropogénico, desarrollo urbano e industrial. Es por ello que esta ecorregión ha sido identificada como una de las 24 áreas prístinas que quedan en el mundo en el siglo XXI (Mittermeier et al. 2003). Para proteger el extremo austral de esta ecorregión se creó la Reserva de la Biosfera Cabo de Hornos (RBCH) con un enfoque de conservación biocultural que consideró los ecosistemas terrestres, marinos y dulceacuícolas y sus interrelaciones (Rozzi et al. 2006a). El trabajo científico de la propuesta de creación de la $\mathrm{RBCH}$ fue liderado por el equipo de investigadores del Parque Etnobotánico Omora (Parque Omora), que constituye el sitio de estudios socio-ecológicos a largo plazo (LTSER) más austral de la Red LTSER-Chile (Rozzi et al. 2010). LTSER es la sigla del nombre en inglés Long-Term SocioEcological Research que utiliza la Red Chilena de Sitios de Estudios Socio Ecológicos a Largo Plazo adscrita a la red mundial International LongTerm Ecological Research (ILTER). Los sitios LTSER ofrecen plataformas idóneas para integrar la investigación científica y la educación (Maass et al. 2016; Holzer et al. 2018). En este sitio, el año 2000 se inició un programa de investigación, educación y conservación biocultural en el extremo austral de Sudamérica (Rozzi et al. 2006b), y luego sus aproximaciones educativas han sido progresivamente incorporadas en otros sitios LTSER de Chile y del mundo (Rozzi et al. 2015). 
En el año 2008 este programa inauguró estudios a largo plazo sobre la diversidad, historias de vida y distribución de insectos dulceacuícolas en la cuenca hidrográfica del río Róbalo, protegida por el Parque Omora (Contador, 2011; Contador et al. 2012, 2014a, 2018; Rendoll et al. 2019). Esta cualidad proporciona una oportunidad única para investigar los efectos del cambio climático sobre una biota que todavía es poco conocida a nivel local y mundial (Contador et al. 2012). Además, en contraste con los invertebrados y ecosistemas dulceacuícolas templados y subpolares del hemisferio norte que han estado sujetos a la lluvia ácida y otros impactos humanos (Hedin et al. 1995), la biota y ecosistemas dulceacuícolas subantárticos todavía se encuentran bien conservados. Sin embargo, esta condición prístina está amenazada por el cambio climático, la homogeneización biocultural y otros procesos del cambio socio-ambiental global que conllevan pérdidas de diversidad tanto biológica como cultural (Rozzi et al. 2010).

A nivel mundial los invertebrados son poco conocidos, valorados $e$ incluso son a menudo considerados molestos o dañinos, pese a su alta diversidad y gran importancia ecológica (Contador et al. 2012; Lockwood, 2013). El conocimiento sobre la diversidad y conservación de los invertebrados es especialmente limitado para los insectos dulceacuícolas, puesto que la mayoría de los estudios se ha realizado sobre insectos terrestres (Contador et al. 2012). Además, existe un marcado sesgo geográfico puesto que los estudios, en su mayoría, se han realizado en el hemisferio norte, especialmente Europa, y eran casi nulos en los ecosistemas subantárticos de Sudamérica hasta el inicio del programa de estudios ecológicos a largo plazo iniciados en el Parque Omora por Contador $y$ colaboradores (2012, 2014a). Sin embargo, estos investigadores se habían enfocado en los cursos de agua, como ríos y esteros, y este trabajo investiga por primera vez la diversidad de invertebrados dulceacuícolas en cuerpos de agua como lagos y lagunas en la $\mathrm{RBCH}$.

En la $\mathrm{RBCH}$, ecólogos, filósofos y artistas del Parque Omora han trabajado en conjunto para comprender la diversidad biocultural local mediante una innovadora aproximación metodológica, la Filosofía Ambiental de Campo (FILAC) (Rozzi et al. 2010). A través de un ciclo de cuatro pasos interrelacionados, esta aproximación integra la investigación científica y filosófica y las artes en la educación y el ecoturismo, contribuyendo a la conservación de la diversidad biocultural. La metodología de la FILAC se ha incorporado en la malla curricular de pre y posgrado de varias universidades chilenas y extranjeras. La tesis tuvo como uno de sus objetivos contribuir a "visibilizar" la singular diversidad de invertebrados dulceacuícolas y sus valores ecológicos, éticos, bioculturales $e$ instrumentales para monitorear el cambio climático.

A nivel mundial, uno de los principales problemas de la investigación científica es su limitada divulgación al público general, la comunidad educativa y los tomadores de decisiones. Esta limitación en la comunicación afecta especialmente a la fauna de invertebrados. A escala global, se conoce muy poco de su estado de conservación. Por ejemplo, de las aproximadamente 1.400 .000 especies conocidas de invertebrados, sólo se ha determinado el estado de conservación para un $1 \%$ de ellas, y la mayoría está clasificada en la categoría de "datos insuficientes" (Collen et al. 2012). Se ha estimado que al menos un $25 \%$ de las especies de insectos descritas a la fecha es vulnerable a procesos de extinción como resultado del impacto antropogénico sobre los ecosistemas (McKinney, 1999). Hallman y colaboradores (2017) han reportado que desde 1990 ha ocurrido una disminución del $75 \%$ en la abundancia de insectos voladores en áreas protegidas de Alemania. Sánchez-Bayo y Wyckhuys (2019) han estimado que, a nivel mundial, más del $40 \%$ de todas las especies de insectos se enfrentará a procesos de extinción en las próximas décadas. Frente a estos escenarios de amenaza, uno de los grandes desafíos para superar esta precariedad sobre el saber del estado de conservación de los insectos es estimular el interés social por ellos, su conocimiento y valoración (Collen et al. 2012; Contador et al. 2012; New \& Samways, 2014). Para enfrentar este desafío, la FILAC ofrece una novedosa herramienta metodológica que es útil para abordar a escala local una problemática que tiene raíces y consecuencias globales. 
Los objetivos de este trabajo son adaptar la metodología de los cuatro pasos de la FILAC para vincular la investigación sobre insectos dulceacuícolas subantárticos con el desarrollo y promoción de actividades educativas formales y no-formales que despierten el interés de la ciudadanía por los insectos y orienten sus percepciones sobre estos organismos, sus hábitats y hábitos de vida. De esta manera, a nivel local se aspira a contribuir a un mayor conocimiento, valoración y conservación de esta singular biodiversidad subantártica, y a nivel global a superar la subvaloración del grupo más diverso de organismos: los insectos.

\section{METODOLOGÍA}

\section{Sitio de Estudio}

La investigación se realizó entre febrero de 2015 y marzo de 2016 en la cuenca hidrográfica del río Róbalo, en la costa norte de la isla Navarino $\left(55^{\circ} \mathrm{S}\right)$ al sur del canal Beagle (Fig. 1). Esta cuenca está protegida por el Parque Etnobotánico Omora (Parque Omora) y provee agua bebestible a Puerto Williams, la ciudad más austral del mundo (Fig. 2). Ubicada en la ecorregión subantártica de Magallanes, la cuenca del río Róbalo recibe las aguas de lluvia más limpias del planeta, puesto que se ubica al sur de las corrientes de vientos que transportan elementos químicos contaminantes (Hedin et al. 1995; Rozzi et al. 2012). Los cursos y cuerpos de agua en esta ecorregión también poseen las concentraciones más bajas de contaminantes inorgánicos registradas en el planeta (Likens, 1991; Mach et al. 2016). La $\mathrm{RBCH}$ protege el extremo austral de esta ecorregión subantártica. Además de una condición prístina, la cuenca del río Róbalo posee un pronunciado gradiente altitudinal $(0-1.000 \mathrm{~m})$ asociado a un gradiente térmico que habilitan a este parque como un laboratorio natural y sitio

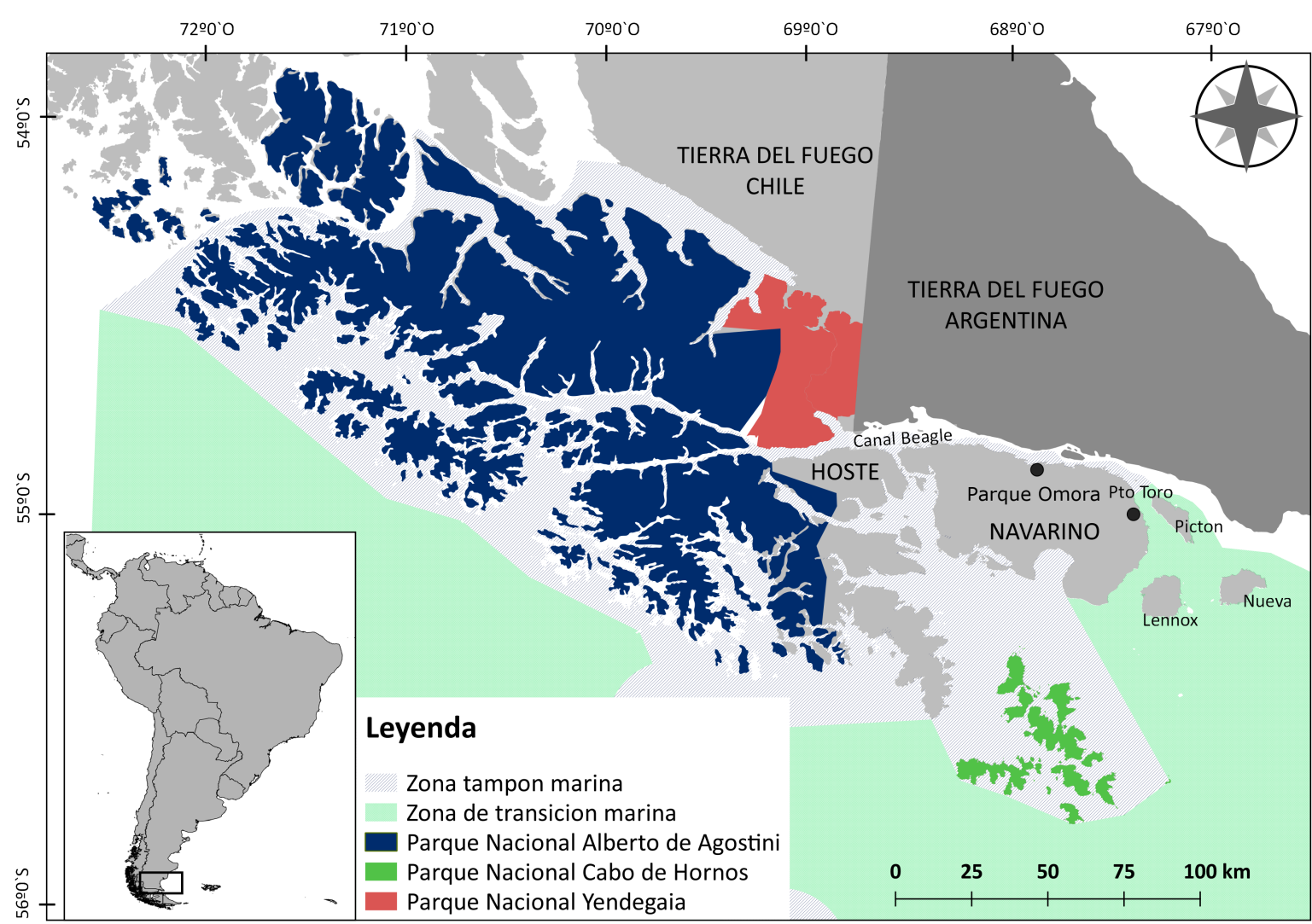

Fig. 1. Ubicación de la Reserva de la Biosfera Cabo de Hornos $(\mathrm{RBCH})$ al sur de Sudamérica, sus parques nacionales y el Parque Etnobotánico Omora, localizados en la isla Navarino. Proyección WGS 84 / UTM 19S, elaboración propia. 


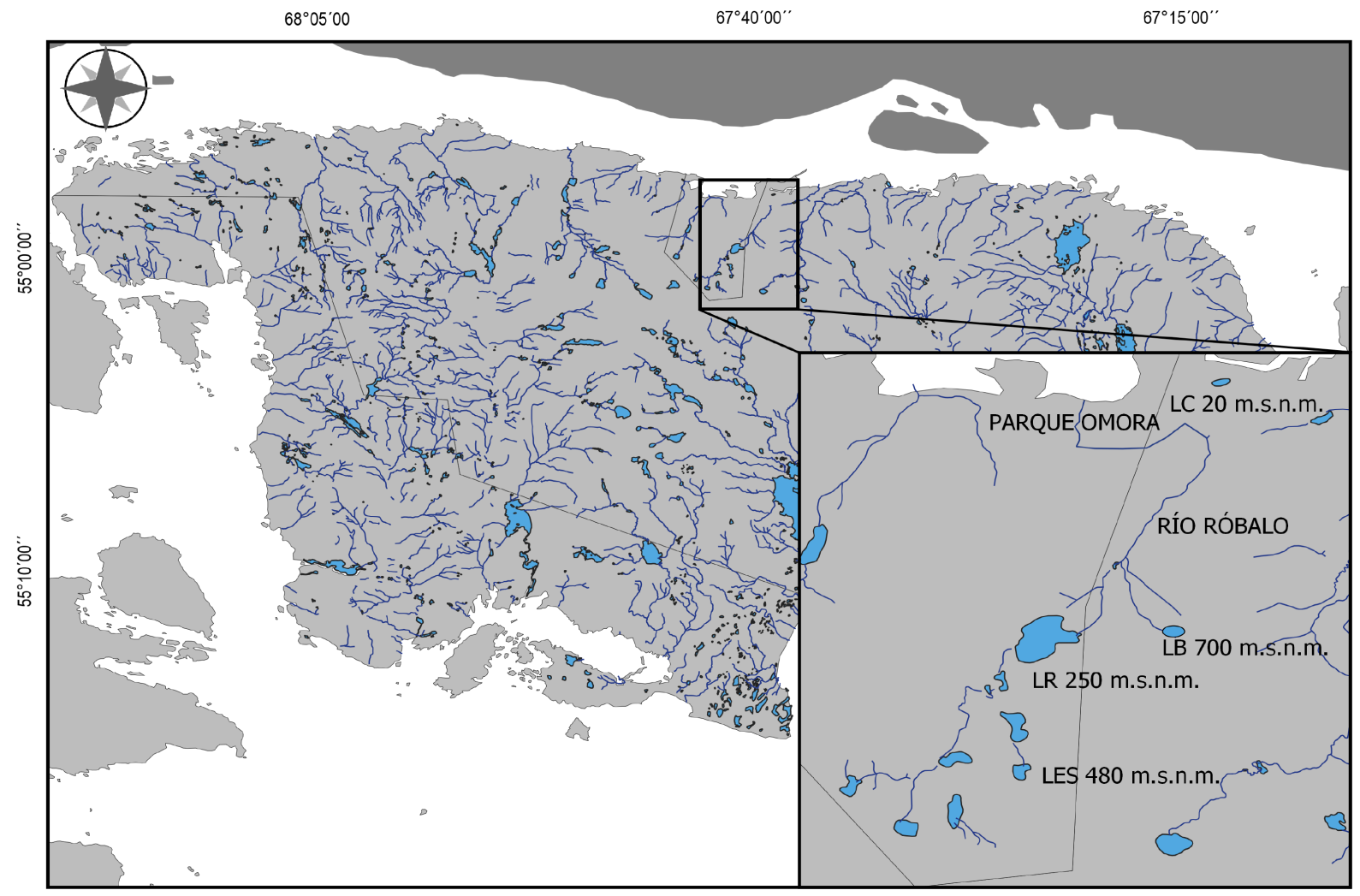

Fig. 2. Isla Navarino y sitios de muestreo en la cuenca hidrográfica del río Róbalo (Rendoll Cárcamo 2018). LC=Laguna Castor; LR=Lago Róbalo; LES=Laguna El Salto;

LB=Laguna Bandera. Proyección WGS 84 / UTM 19S, elaboración propia.

de estudios socio-ecológicos a largo plazo ideal para investigar los efectos del cambio climático sobre los ecosistemas dulceacuícolas y sus biotas (Contador et al. 2015). También, el parque posibilita generar actividades y materiales de educación, comunicación y conservación de la biodiversidad, incluyendo a los organismos poco percibidos y valorados, como los insectos dulceacuícolas (Contador et al. 2014a).

El estudio de los insectos dulceacuícolas a lo largo del gradiente altitudinal de la cuenca hidrográfica del río Róbalo, se realizó en cuatro lagunas ubicadas en cuatro pisos altitudinales (Fig. 2): 20 msnm en una matriz de bosques siempreverde (dominados por Nothofagus betuloides y Drymis winteri) y turberas de Sphagnum sp.; $250 \mathrm{msnm}$ en una matriz de bosque mixto deciduo y siempreverde (dominados por $N$. betuloides y $N$. pumilio) y matorrales; 480 msnm en bosques deciduos achaparrados en formación de krummholz (dominados por
$N$. pumilio y $N$. antarctica), y $700 \mathrm{msnm}$ en ecosistemas altoandinos subantárticos dominados por plantas en cojín, líquenes y musgos.

\section{El Ciclo de Cuatro Pasos de la Filosofía Ambiental de Campo (FILAC)}

El trabajo de investigación, educación y conservación se basó en la FILAC y su ciclo de cuatro pasos, que son: i) investigación interdisciplinaria ecológica y filosófica, ii) creación de metáforas y/o narrativas simples, iii) diseño de actividades guiadas con una orientación ética y ecológica, y iv) implementación de áreas de conservación in situ.

Paso 1. Investigación interdisciplinaria ecológica y filosófica

La investigación ecológica se enfocó en los ecosistemas dulceacuícolas y su diversidad de macroinvertebrados. Los microhábitats se 
caracterizaron de acuerdo a sus atributos físicos y bióticos. Trimestralmente, en cada estación del año se midieron los parámetros químicos del agua con sensores multiparámetro (YSI 605 595 Professional Plus multimeter y ConductivitypH-TDS Hanna Tester HI98130) y se registró la temperatura, el pH y la conductividad en 3 ocasiones para cada microhábitat en cada evento de muestreo. Además, para estudios a largo plazo de la temperatura del agua, se instalaron en cada laguna (a unos $20 \mathrm{~cm}$ de profundidad y en un punto al azar) instrumentos $(\mathrm{HOBO} \AA$ data loggers, model U22 Water Temperature Pro version 2) que han realizado registros cada 4 horas desde marzo de 2015 hasta el presente.

Los atributos bióticos consideraron la composición del sustrato, la presencia/ausencia $y$ tipo de vegetación en las zonas ribereñas $y$ en los fondos de cada laguna: rocosos (gravas desde $1 \mathrm{~cm}$ a $10 \mathrm{~cm}$ ), vegetación sumergida (plantas vasculares acuáticas o sumergidas), debris (hojarasca, ramas, raíces y/o troncos muertos) y musgos acuáticos (total o parcialmente sumergidos) (Rendoll Cárcamo, 2018). Durante el año 2015 se colectaron macroinvertebrados acuáticos durante el verano austral, otoño $y$ primavera, y en cada microhábitat se tomaron tres muestras con una red de muestreo en forma de "D" (malla de $150 \mu \mathrm{m}$ ), durante 3 minutos (1 min para cada muestra). Cada muestra se almacenó en $70 \%$ de etanol, fueron etiquetadas y transportadas al laboratorio para su procesamiento donde los macroinvertebrados fueron identificados hasta el nivel taxonómico más detallado posible. Además, en base a una revisión bibliográfica (Fernández \& Domínguez, 2001; Merritt \& Cummins, 2008), los macroinvertebrados fueron clasificados de acuerdo a grupos funcionales de alimentación: colector-recolector, filtrador, depredador, triturador, raspador y herbívoro.

La investigación filosófica se enfocó en el análisis de un ensayo fundacional de la ética ambiental, Las raíces históricas de nuestra crisis ecológica, originalmente publicado como The Historical Roots of Our Ecological Crisis en la revista Science el año 1967 por el historiador de la ciencia estadounidense Lynn White Jr. Se examinó este ensayo considerando interpretaciones recientes (Castro, 2007; Hargrove, 2007; Rozzi, 2007), se complementó con el marco conceptual de las 3Hs (hábitats, hábitos de vida y co-habitantes) de la ética biocultural (Rozzi et al. 2012) y con la discusión sobre la falta de conocimiento y valoración por los invertebrados (Cardoso et al. 2011).

\section{Paso 2. Creación de metáforas y/o narrativas simples}

Para integrar los resultados de la investigación con la educación ambiental no formal y promover un respeto por la entomofauna dulceacuícola de la $\mathrm{RBCH}$, se trabajó en la composición de metáforas. Este paso tiene una doble intención. Por un lado, facilitar un diálogo con el público y, por otro, integrar los resultados del Paso 1 a través del pensamiento analógico. Las metáforas contribuyen a sintetizar conceptos, hechos, valores, acciones de educación y conservación biocultural (Rozzi et al. 2010). El concepto de complejidad ecológica ha emergido de manera prominente (Bradbury et al. 1996) y "las metáforas pueden alterar y difundir significados a través y más allá de los límites de un discurso especializado" (Proctor \& Larson, 2005).

Paso 3. Diseño de actividades guiadas con una orientación ética y ecológica

En este trabajo consideramos que, como plantea la FILAC, los encuentros directos con los seres vivos en sus hábitats son esenciales para comprender y valorar la diversidad biocultural. Nos enfocamos en los encuentros con los ecosistemas dulceacuícolas y sus invertebrados que pueden ser comprendidos como co-habitantes. Así, no sólo conceptual sino también experiencialmente se pueden percibir las interrelaciones vitales entre los hábitats y hábitos de vida de los co-habitantes (humanos y otros-quehumanos). De esta manera, además de transformar las formas de conocer, también se orientan formas de cohabitar en los ecosistemas.

Paso 4. Implementación de áreas para la conservación biocultural in situ

Este trabajo se enfocó en propuestas para la protección y valoración de la cuenca hidrográfica del río Róbalo y sus co-habitantes. Esta acción es relevante por al menos tres razones: i) protege 
hábitats y habitantes nativos y sus hábitos (interacciones ecológicas), ii) permite a los visitantes observar y disfrutar estos hábitats e interacciones, $y$ iii) fomenta el compromiso ético y ecológico con el cuidado de la diversidad de hábitats y sus diversas formas de vida, incluyendo organismos poco valorados socialmente hasta el momento, tales como los insectos dulceacuícolas.

\section{RESULTADOS Y DISCUSIÓN}

En la metodología de la FILAC el propósito del Paso 1 en el ciclo de cuatro pasos es integrar la investigación ecológica con la investigación filosófica, y en los siguientes integrar esta investigación con actividades educativas y de conservación biocultural. A continuación, presentamos los resultados y discusión en cada uno de los pasos del ciclo de la FILAC.

\section{Paso 1. Investigación Interdisciplinaria Ecológica y Filosófica}

En base a la investigación ecológica se determinó que las características físicoquímicas de los ecosistemas dulceacuícolas varían estacionalmente y a lo largo del gradiente altitudinal. La temperatura del agua de las lagunas presentó marcadas variaciones altitudinales y estacionales, con períodos de congelamiento y temperaturas cálidas (Rendoll et al. 2019). La laguna cercana a nivel del mar tuvo una temperatura del agua promedio de $16,99^{\circ} \mathrm{C}$ en el mes más cálido (diciembre) y de $-0,46^{\circ} \mathrm{C}$ en el mes más frío (julio). En la laguna ubicada a mayor altitud $(700 \mathrm{~m})$ los valores registrados en estos meses fueron de $14,70^{\circ} \mathrm{C}$ y de $-1,56^{\circ} \mathrm{C}$ (Rendoll Cárcamo, 2018). La acidez y la conductividad del agua también disminuyeron significativamente con la altitud de las lagunas. Los promedios anuales tuvieron valores de aguas ácidas 5,97 $\mathrm{pH}$ (potencial de hidrógeno) y alta conductividad $147,3 \mu \mathrm{S} / \mathrm{cm}$ en la laguna más baja y de aguas alcalinas 7,61 pH y baja conductividad $49,5 \mu \mathrm{S} / \mathrm{cm}$ en la laguna de mayor altitud (Rendoll et al. 2019).

Se encontró que la diversidad taxonómica de insectos y otros macroinvertebrados dulceacuícolas disminuyó significativamente con la altitud de las lagunas. En total se recolectaron 8.435 individuos, entre los que identificamos 28 taxa pertenecientes a tres filos: Annelida, Arthropoda y Mollusca. Annelida incluyó tres taxa de la clase Clitellata, incluyendo dos órdenes: Hirudinae y Oligochaeta. Mollusca incluyó dos taxa de las clases Bivalvia y Gastropoda, incluyendo los órdenes Veneroida e Hygrophila, respectivamente. El filo de los artrópodos incluyó 23 taxa de tres clases: Malacostraca, Branchiopada e Insecta (Hexapoda). Las dos primeras clases incluyeron un taxón cada una pertenecientes a los órdenes Amphipoda y Cladocera, respectivamente. La clase de los insectos fue la más diversa e incluyó 21 taxa, pertenecientes a los órdenes Odonata (1 taxón), Ephemeroptera (2 taxa), Coleoptera (2 taxa), Hemiptera (1 taxón), Diptera (10 taxa), Trichoptera (3 taxa) y Plecoptera (2 taxa) (Rendoll Cárcamo, 2018; Rendoll et al. 2019). El detalle de la composición taxonómica de macroinvertebrados dulceacuícolas en cada laguna ha sido descrito previamente (Rendoll Cárcamo, 2018; Rendoll et al. 2019) y el análisis en este trabajo se centrará en insectos dulceacuícolas con el mayor valor potencial para actividades educativas.

Entre los insectos dulceacuícolas dos grupos llamaron la atención por su relevante papel ecológico y valor comunicacional para los estudiantes y la comunidad general: las moscas (Diptera) de la familia Chironomidae, distribuidas a lo largo del gradiente altitudinal, y el escarabajo depredador Lancetes angusticollis, conocido como tigre del agua. El número de generaciones por año de los insectos es mayor bajo temperaturas más altas. Por lo tanto, los quironómidos podrían servir de indicadores ideales para la temperatura del agua de lagunas a distintos niveles altitudinales. El coleóptero depredador fue abundante en la laguna del nivel altitudinal más bajo y, por lo tanto, de más fácil acceso para la realización de actividades educativas.

Para identificar los obstáculos y superar el desconocimiento sobre los invertebrados con los estudiantes de Puerto Williams y otros miembros de la comunidad, los siete impedimentos para la conservación de invertebrados y cómo abordarlos propuestos por Pedro Cardoso y colaboradores (2011) proveyeron un marco que permitió identificar barreras cognitivas que han sido poco resaltadas. A continuación se detallan estos impedimentos: (1) los invertebrados y sus roles ecosistémicos 
son prácticamente desconocidos para el público en general (el dilema público); (2) la mayoría de los encargados de formular políticas y las partes interesadas desconocen los desafíos/estados de conservación de invertebrados (el dilema político); (3) la ciencia básica sobre invertebrados es escasa y no tiene fondos suficientes (el dilema científico); (4) la mayoría de las especies no están descritas (el déficit de Linneo); (5) la distribución geográfica de las especies descritas es mayoritariamente desconocida (el déficit de Wallace); (6) se desconoce la abundancia de especies y sus cambios en el espacio y el tiempo (el déficit de Preston); (7) las formas de vida de las especies y la sensibilidad a cambios en sus hábitats son en gran medida desconocidas (el déficit de Hutchinson).

El estudio de Javier Rendoll Cárcamo (2018) determinó que los invertebrados dulceacuícolas son indicadores sensibles de variaciones térmicas, que algunas especies cumplen funciones tróficas clave, que la composición de especies en las lagunas varía en el espacio y el tiempo, y que sus sensibilidades a los cambios en el hábitat son muy poco conocidas. Estos hallazgos se relacionan con los impedimentos del dilema público, del déficit de Preston y del déficit de Hutchinson.

A partir del análisis de textos filosóficos, sin embargo, podemos interpretar que el déficit de Hutchinson va más allá del ámbito puramente ecológico y abarca un ámbito ético en la valoración de los invertebrados.

Respecto a la dimensión ética, Rozzi (2007) destaca tres tesis centrales que fundamentan el ensayo de Lynn White Jr. (1967) sobre los orígenes de la crisis ambiental global. Primero, que la victoria del cristianismo sobre el animismo pagano representa una revolución psíquica en la historia de la cultura occidental. Segundo, que el "credo baconiano", del filósofo inglés Francis Bacon (1561-1626) quien propuso que la misión de la ciencia era controlar la naturaleza para el progreso de la humanidad, representa una de las mayores revoluciones civilizatorias en la historia de la humanidad desde la invención de la agricultura. Este credo se ha consolidado con la adopción de la tecnología como herramienta de dominio sobre la naturaleza y norma conductual prevaleciente en la sociedad actual. Tercero, que para confrontar esta insensible relación de explotación se requieren transformaciones éticas en la forma de ver, sentir y valorar los ecosistemas, sus co-habitantes humanos y no humanos, y las interacciones entre ellos y su entorno.

De acuerdo a White Jr. (1967), el advenimiento del cristianismo en reemplazo del animismo pagano, la noción bíblica que el ser humano fue creado a semejanza de Dios, ha continuado arraigado en la sociedad moderna y ha justificado la explotación indiferente de la naturaleza y sus seres vivos. Propone que, en contraste, las culturas pre-cristianas creyeron y respetaron a los espíritus guardianes que habitaban en los árboles, ríos, bosques, montañas y otros seres. Estos espíritus eran distintos a los seres humanos y debían ser apaciguados previamente a la extracción de un recurso. El cristianismo sustituyó esta creencia por un culto a espíritus que habitan exclusivamente en el cielo, o en la tierra confinados a los seres humanos. Esta desaparición de una restricción en las creencias dejó libre el campo para la explotación de recursos naturales. White Jr. (1967, p. 83) concluye que "el cristianismo es la religión más antropocéntrica que el mundo ha conocido, en particular en su forma occidental".

Los espíritus en los objetos naturales, quienes en un principio habían protegido a la naturaleza de la acción del hombre, se esfumaron. El monopolio efectivo del hombre sobre el espíritu en este mundo fue confirmado y las antiguas inhibiciones para explotar la naturaleza desaparecieron.

Desde la ética biocultural, Rozzi (2013) ha criticado a Lynn White Jr. por su análisis eurocéntrico puesto que otras cosmovisiones, paralelas al cristianismo, han mantenido $y$ continúan manteniendo la presencia de espíritus en los seres vivos y ecosistemas. Por ejemplo, el pueblo lenca en Centroamérica defiende hoy activamente los espíritus guardianes de sus ríos. Una de sus lideresas indígenas y ecofeministas, Berta Cáceres, fue recientemente asesinada por defender esta creencia y la conservación de los ríos (May, 2018). Dentro de Europa destaca la cultura celta, que cree que cada montaña, río, arroyo, árbol, rocas y otros seres están habitados por espíritus, que refrenan la explotación. Respecto al río Róbalo y otros ríos de la RBCH, Contador y colaboradores (2018, p. 194) han documentado que Julia González, artesana de la Comunidad Indígena Yagán que hoy 
habita en la isla Navarino, relata que "los espíritus de los antepasados yaganes viven entre las rocas de los ríos, y que cuando una persona duerme por primera vez cerca del lecho de un río, puede escucharlos hablar e incluso bromear".

Desde múltiples cosmovisiones que nutren e informan una ética biocultural, el río es un hogar para muchas formas de vida donde la vida humana trasciende y se mantiene en el tiempo, formando parte del ecosistema ribereño. El río puede ser concebido como "una comunidad de vida que aloja también a los antepasados y seres queridos que han fallecido" (Contador et al. 2018, p. 194). Por lo tanto, existen sensibilidades no sólo en los invertebrados dulceacuícolas que son poco conocidas, sino también en las distintas culturas que valoran a estos invertebrados. Estas múltiples formas de valoración deben recuperarse e incluirse en la educación para cultivar un respeto por los ecosistemas dulceacuícolas, los invertebrados, los seres humanos y otros seres que co-habitan con hábitos de vida contrastantes en estos hábitats singulares.

Paso 2. Composición de Metáforas: "Insectos Acuáticos, Centinelas Invisibles"

El término metáfora proviene de los términos griegos meta (=más allá) y pherein (=transferir) que indican el traslado de un concepto por otro. Estas figuras del lenguaje surgen desde un pensamiento analógico que permite comprender la realidad (Rozzi et al. 2010) y, por lo tanto, las metáforas son hoy consideradas un componente constitutivo en la ciencia, facilitando la comprensión de temas abstractos y complejos, y la vinculación de diversas disciplinas (Proctor \& Larson, 2005).

En este trabajo compusimos la metáfora "insectos acuáticos, centinelas invisibles" con el doble propósito de comprender y comunicar el papel clave que los invertebrados cumplen en los ecosistemas dulceacuícolas, pero que es prácticamente desconocido para el público en general. Esta metáfora permite abordar el dilema público planteado por Cardoso y colaboradores (2011). Además, destaca el valor instrumental, es decir, la utilidad que estos invertebrados tienen para la sociedad humana en cuanto actúan como centinelas de un problema apremiante: el cambio climático global.
En términos más amplios, la metáfora permite apreciar el papel de bioindicadores de los insectos acuáticos (Dallas \& Rivers-Moore, 2012; Rendoll Cárcamo, 2018). El carácter invisible estimula la toma de conciencia acerca de que los insectos (y otros invertebrados) y sus roles ecosistémicos son poco conocidos y subvalorados en estrategias de conservación y la cultura cotidiana. Dentro de la ciencia, la bibliografía centrada en la utilidad de los invertebrados como bioindicadores supera en órdenes de magnitud a la bibliografía dedicada a su estado de conservación (Contador et al. 2012; Ramírez \& Gutiérrez-Fonseca, 2014). El adjetivo invisible cataliza una ruptura de paradigmas científicos y de la conservación centrados en la fauna de vertebrados; además, invita a los estudiantes y otros ciudadanos a la acción de observar, indagar y conocer, que parafraseando al biólogo de la conservación Daniel Janzen es un paso necesario para la conservación, "si no lo conoces, no lo puedes amar, y si no lo amas, no puedes salvarlo" (Cardoso et al. 2011, p. 2650).

\section{Paso 3. Actividades de Campo: Explorando} la Ecología de los Tigres de Agua

La metáfora compuesta en el Paso 2 invita a conocer a través de "encuentros directos" con los invertebrados dulceacuícolas, como ha sido propuesto anteriormente (Contador, 2011). La finalidad de esta nueva metáfora es contribuir a cambiar la percepción social prevaleciente hacia los insectos, invitando a reconocer en terreno la utilidad de estos organismos como centinelas para alertar sobre las consecuencias del cambio climático global. Además, la expresión "insectos dulceacuícolas" vincula hábitats poco explorados (los ecosistemas dulceacuícolas) con co-habitantes poco conocidos (insectos) que despliegan múltiples funciones ecológicas y hábitos de vida únicos por su singularidad y belleza.

La actividad de campo diseñada en este Paso 3, "Explorando la ecología de los tigres de agua", procura recuperar una práctica naturalista que favorece el reconocimiento en terreno de los vínculos vitales entre la conservación de los hábitats y los hábitos de vida de los co-habitantes (humanos y otros-que-humanos). Este marco conceptual de las 3Hs de la ética biocultural (Rozzi, 2013) 
orienta ecológica y éticamente la observación de comportamientos (o hábitos) como el registrado en escarabajos dulceacuícolas (co-habitantes) en las lagunas (hábitats) de la cuenca del río Róbalo. Basado en un pensamiento analógico, esta actividad establece una comparación entre el papel de feroces depredadores que cumplen los tigres en ecosistemas terrestres y este escarabajo en los ecosistemas dulceacuícolas. Ambas conductas pueden ser observadas en terreno, pero con menor nivel de riesgo en la cuenca del río Róbalo.

Es notable que los nombres científicos del orden y de la familia a la que pertenece este escarabajo depredador vinculen sus hábitos de vida con los hábitats que hacen posibles estos hábitos. Los escarabajos pertenecen al orden Coleoptera que describe que las alas (en griego=pteron) están guardadas dentro de una caja (en griego=koleos), que es su propio cuerpo, y la especie Lancetes angusticollis, que es el tigre de agua que habita en las lagunas de la isla Navarino, pertenece a la familia Dytiscidae que describe el hábito de bucear (en griego=dytikos).

Con estos conceptos y actividades de campo los estudiantes del Liceo Donald McIntyre Griffiths (DMG), único establecimiento escolar en
Puerto Williams, se motivaron por la estética y los hábitos de los tigres de agua. Investigaron en terreno y laboratorio el ciclo larval de Lancetes angusticollis, y presentaron su trabajo en la 45a Feria Científica Juvenil del Museo Nacional de Historia Natural (Fig. 3). Esta fue la primera participación de alumnos del Liceo DMG en la mencionada feria y contribuyó a comunicar un hábito depredador, con distintas presas y conductas de caza poco conocidas. Para conducir en terreno la experiencia de "Explorando la ecología de los tigres de agua" puso especial atención en tres actividades de campo.

\section{La observación del hábitat}

Las lagunas, al igual que los ríos, poseen diferentes microhábitats y en ellos habitan distintos invertebrados. Los Dytíscidos son depredadores y cazadores eficaces, generalmente se les encuentra entre la vegetación sumergida (vascular y/o novascular) y dependiendo de la estación es posible encontrar también a sus larvas, conocidas como tigres de agua. En esta etapa, los participantes pueden asociar el hábitat y hábitos de los animales que allí co-habitan.

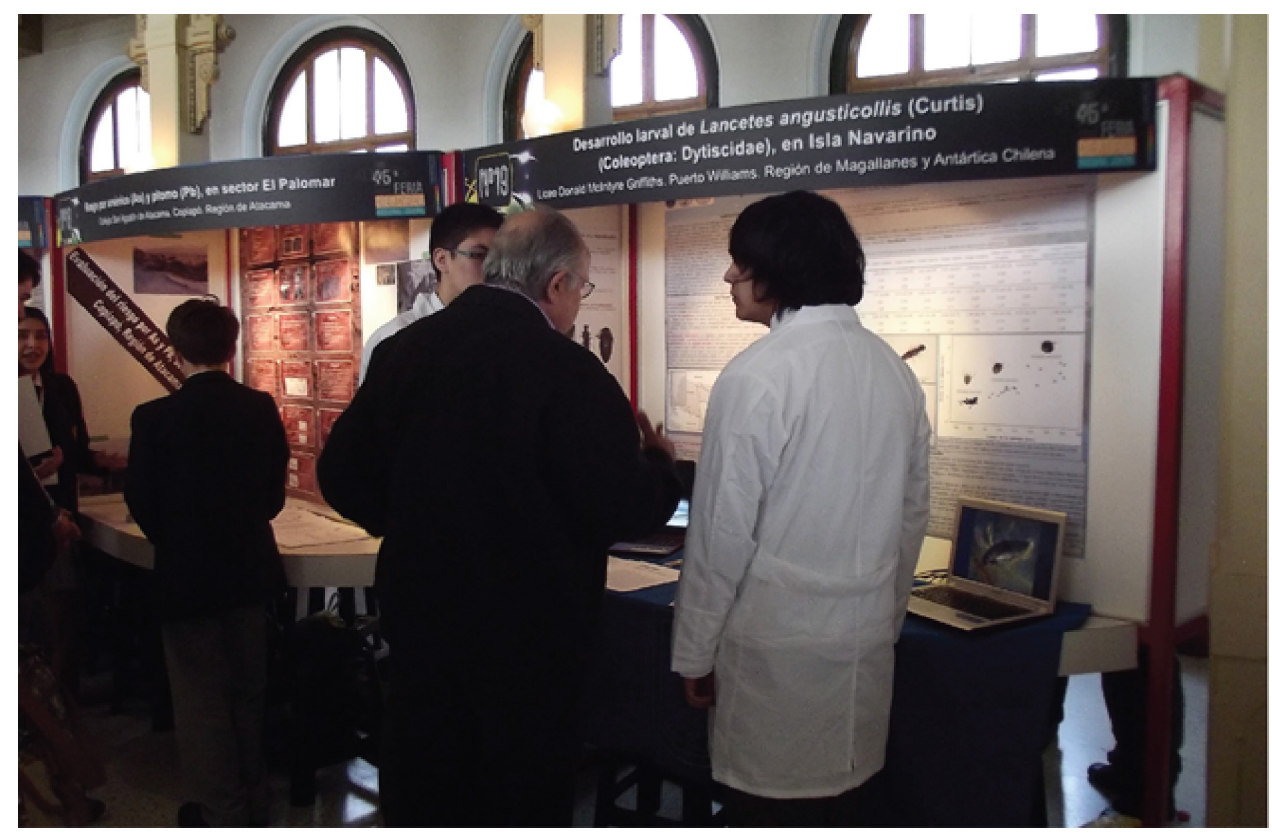

Fig. 3. Estudiantes del Liceo Donald McIntyre Griffiths en el Museo Nacional de Historia Natural (MNHN) en Santiago. En la foto, los alumnos Alex Neira y Rodrigo Soto explican su trabajo a un directivo del MNHN. Profesor y asesor científico: Javier Rendoll Cárcamo. 


\section{Encuentros directos con coleópteros}

Con una pequeña red se colectan algunos individuos, luego se colocan en una fuente con agua de la misma laguna y los estudiantes observan su comportamiento. Se dibuja la morfología de L. angusticollis y se comprende cómo las distintas partes de su cuerpo cumplen funciones específicas. Por ejemplo, el tercer par de patas de estos coleópteros posee setas largas y gruesas que actúan a modo de remos que utilizan como mecanismo de propulsión. También es posible observar que estos insectos suben a la superficie a capturar burbujas de aire que les permiten respirar bajo el agua, y para lograr dicha captura los Dytíscidos poseen pequeños pelos "hidrófugos" en la parta baja del abdomen. Con un fin educativo, se puede depositar una larva de díptero en la fuente de agua con el propósito de estimular y observar el comportamiento cazadordepredador del coleóptero.

\section{Regresar a los Dytíscidos a su hábitat}

Luego de las actividades de observación y encuentros directos, cada participante debe regresar los coleópteros a su hábitat. Esta acción fomenta un respeto tanto con los insectos como con sus hábitats y comunidades de co-habitantes, bajo una comprensión de los vínculos vitales entre ellos. Contador (2011) ha destacado el valor que esta acción tiene para cambiar la percepción prevaleciente en la ciencia, que considera a los insectos como objetos de estudio, y reconocerlos más bien como sujetos, es decir, formas de vida otra-que-humana que tienen un fin en sí mismas y a la vez co-habitan con los seres humanos. Rozzi (2015) ha propuesto que la teoría biológica de la unidad de la vida constata que los invertebrados tienen mecanismos de respiración celular muy similares a los seres humanos, y en griego el término espíritu proviene del verbo respirar. Por ende, tanto los seres humanos como los insectos tendrían espíritu, superando el dualismo criticado por Lynn White Jr. (1967).

Con fines educativos más amplios, los insectos han sido considerados principalmente como modelos útiles para enseñar principios biológicos generales, y a menudo los científicos han involucrado tempranamente a jóvenes en procesos de descubrimiento (Fischang, 1976; Ernst et al. 2013). Estas primeras experiencias involucran encuentros directos, observaciones y cuestionamientos que estimulan el pensamiento crítico y la formación de hipótesis (Ernst et al. 2013). Estas actividades ofrecen oportunidades de enseñanza que causan impresiones en los estudiantes, que además pueden ser compartidas con la comunidad (Ernst et al. 2013). La inclusión de alumnos y otros miembros de la comunidad en las actividades científicas permite que los programas de conservación contribuyan a superar los déficits de percepción (Cardoso et al. 2011) y la falta de conocimiento sobre los insectos (Samways, 2015).

Paso 4. Implementación de áreas para la conservación biocultural in situ

Los investigadores del Parque Omora han implementado un sistema de senderos $y$ estaciones para la realización de actividades de educación ambiental y ecoturísticas al aire libre (Rozzi et al. 2008, 2010). Estos senderos y estaciones permiten, mediante la observación in situ, presentar a los estudiantes y otros visitantes los ecosistemas (hábitats) y las interrelaciones entre sus co-habitantes y hábitos de vida (Rozzi et al. 2008). Dentro del área del parque destinada a las actividades de educación y turismo no se consideraban lagunas. Con el objetivo de reparar esta carencia, en este trabajo se identificaron lagunas dentro de $y$ aledañas al parque que pueden ser visitadas con fines educativos. Aprovechando las variaciones en marcados gradientes altitudinales en la isla Navarino (Fig. 4, Contador, 2011; Contador et al. 2015; Rendoll Cárcamo, 2018), en este trabajo se propusieron cuatro lagunas que permiten observar diferentes formaciones vegetacionales, con cambios en la riqueza, abundancia y composición de las comunidades de invertebrados dulceacuícolas.

Estación 1: Laguna Castor. Su nombre proviene del hecho que esta laguna está perturbada por una castorera, construida por el castor americano (Castor canadensis), un 


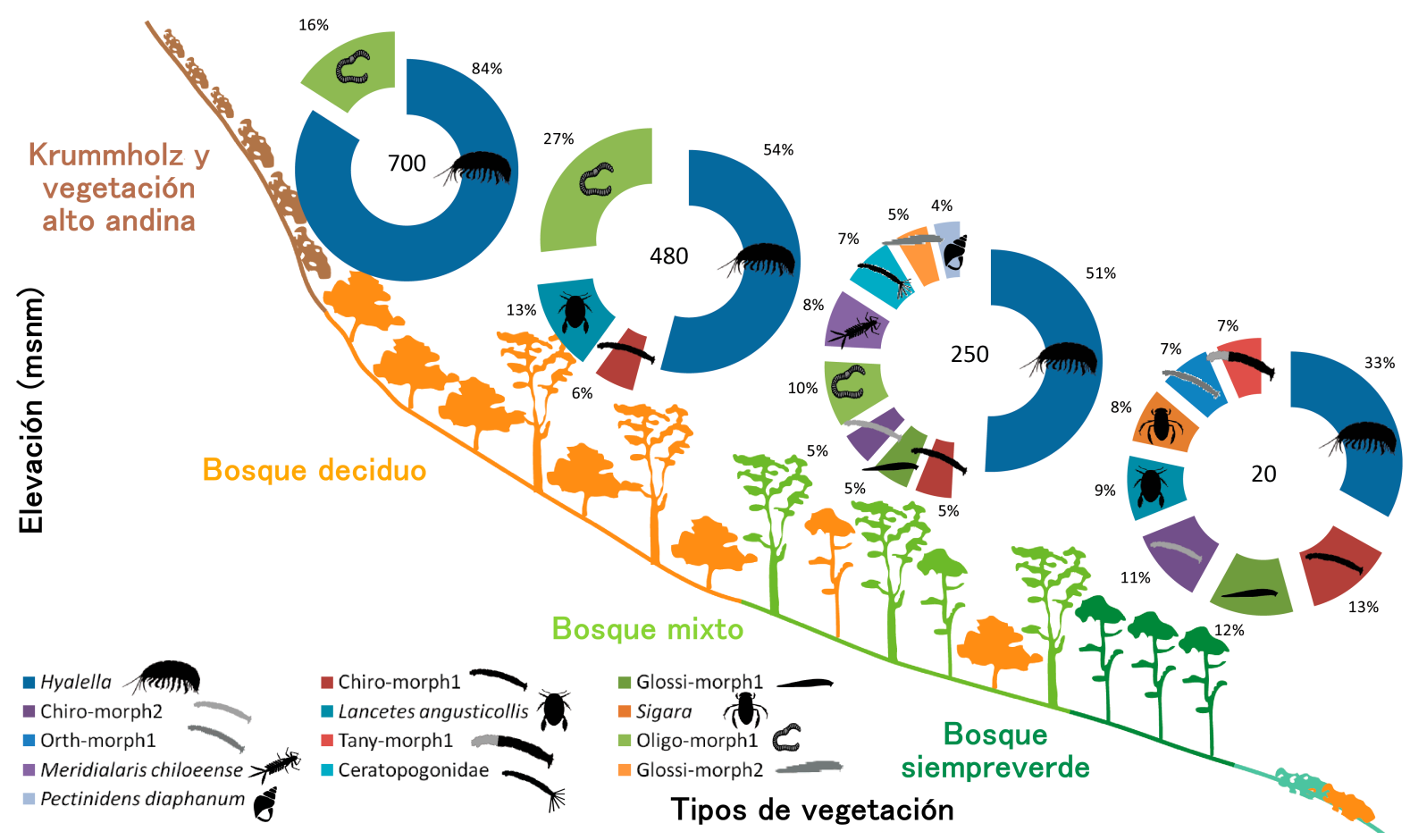

Fig. 4. Esquema del gradiente altitudinal de la cuenca hidrográfica del río Róbalo. Se indica la elevación de cada una de las lagunas estudiadas junto con la composición del ensamble, destacando el depredador Lancetes angusticollis (Coleoptera) y la familia de los quironómidos (Diptera). (Figura modificada de Contador et al. 2014b y Rendoll et al. 2019).

roedor exótico introducido en la isla Navarino a mediados del siglo XX (Schüttler et al. 2019). Se encuentra aledaña al Parque Omora cercana al nivel del mar, y sus riberas están dominadas por bosques siempreverde de coigüe de Magallanes ( $N$. betuloides) y canelo (D. winteri) inmersos en una matriz de turbera dominada por Sphagnum magellanicum. En esta laguna se registró la mayor riqueza de invertebrados dulceacuícolas, y ofrece un lugar ideal para "explorar la ecología de los tigres de agua" (Lancetes angusticollis). Además, se puede apreciar la diversidad de hábitos de vida de otros coleópteros acuáticos (Liodessus sp.), libélulas (Rhionaeschna variegata), y pulgas de agua de la familia Daphniidae.

Estación 2: Lago Róbalo. Es el mayor de los cuerpos de agua de la cuenca del río Róbalo. Se ubica dentro del parque a una altitud de 250 msnm, y sus riberas están dominadas por bosques mixtos siempreverde ( $N$. betuloides) y deciduos de lenga ( $N$. pumilio), formaciones de matorral y turberas en las zonas planas. Este lago presenta un sustrato rocoso con bolones de gran tamaño entre los cuales se encuentra gran cantidad de troncos y ramas transportados por castores desde los bosques ribereños. En este lago se pueden observar insectos que pueden utilizarse como bioindicadores del calentamiento global. Los estudiantes comprenden así este valor utilitario o instrumental de los "insectos acuáticos como centinelas invisibles" del cambio climático. Estos insectos se hacen visibles para los estudiantes que pueden observar especialmente tricópteros y efímeros que sorprenden con sus hábitos de vida, cuando en el verano presentan grandes enjambres de adultos apareándose.

Estación 3: Laguna El Salto. Presenta una hermosa cascada en la parte alta del río Róbalo y se ubica a $480 \mathrm{msnm}$, cerca del límite arbóreo. Sus riberas están rodeadas por bosques achaparrados, en una matriz de sustratos rocosos y plantas en forma de cojín (Méndez et al. 2013). En la laguna prevalece el sustrato rocoso y crecen grandes parches de musgos acuáticos y subacuáticos, vegetación 
vascular sumergida y abundantes raíces y ramas de árboles. Esta laguna es especialmente apropiada para observar la diversidad de microhábitats, hábitos de vida y co-habitantes. Los co-habitantes invertebrados incluyen al "tigre del agua" junto a abundantes anfípodos (Hyalella sp.), sanguijuelas (Glossiphoniidae), larvas de moscas (o dípteros) de la familia Chironomidae y miembros de otro filo animal: los moluscos, representados por el pequeño bivalvo Pisidium magellanicum (Sphaeriidae) que habita en los sustratos arenosos bajo restos vegetales.

Estación 4. Laguna Bandera. Su nombre proviene del hecho que esta laguna está sobre el cerro Bandera. Se ubica a $700 \mathrm{~m}$ en las formaciones altoandinas subantárticas. La vegetación circundante está compuesta por líquenes, musgos y plantas vasculares del género Nassauvia, que crecen tanto en el ambiente terrestre como en la interfase tierraagua y algunas plantas están completamente sumergidas (Rendoll Cárcamo, 2018). Esta laguna se congela durante siete a ocho meses cada año. Los estudiantes y otros visitantes pueden apreciar los hábitos de vida de anfípodos y quironómidos, y ocasionalmente plecópteros oligotermos (=tolerantes a las bajas temperaturas) de la familia Gripopterygidae, que están adaptados a condiciones extremas.

\section{COMENTARIOS FINALES}

Cada una de las cuatro estaciones permite implementar pequeñas salas de clase al aire libre, donde los insectos acuáticos "invisibles" comienzan a ser visibles. Los encuentros directos con estos co-habitantes invertebrados poco conocidos, y la comprensión de sus relaciones ecológicas con diversos hábitats y hábitos de vida, sorprenden a los visitantes y fomentan una valoración por los insectos dulceacuícolas.

Este trabajo diversifica el programa de investigación, educación yecoturismo sumergidos con lupa iniciado por Contador y colaboradores (2014a, b), que estimuló acciones para la apreciación y conservación de los invertebrados dulceacuícolas. Los estudios iniciales de Contador y colaboradores (2018) se centraron en cursos de agua $e$ insectos constructores de microhábitats, como los tricópteros. La investigación de Rendoll y colaboradores (2019) complementó las realizadas anteriormente al centrarse en cuerpos de agua y en insectos con un papel ecológico clave: el de depredadores, tales como "el tigre del agua".

La metodología de la FILAC permite que las prácticas educativas no sean homogeneizantes sino que descubran, comprendan y valoren las singularidades de los hábitats, hábitos de vida y diversidad de co-habitantes en cada localidad. La Fig. 5 ilustra una síntesis de los cuatro pasos de la FILAC adaptada en este trabajo. Las actividades educativas que incluyen insectos proporcionan una plataforma para el entrenamiento y el pensamiento científico desde etapas escolares tempranas (Ernst et al. 2013). Se espera que las futuras generaciones refuercen los estudios ecológicos sobre insectos en la $\mathrm{RBCH}$. Para ello es necesario fortalecer la difusión de resultados científicos, la integración de la comunidad local y potenciar la educación ambiental formal y no-formal.

Este trabajo ha integrado la investigación ecológica con una investigación filosófica en una innovadora aproximación educativa para conocer, valorar y conservar a los invertebrados dulceacuícolas. Tanto en la ecorregión subantártica como en otras regiones del planeta, los invertebrados, particularmente los insectos, son los organismos más diversos. Sin embargo, como se señaló antes, son poco conocidos y valorados. De esta manera, desde el sur del mundo hacemos un aporte que puede tener aplicaciones globales, especialmente en otros sitios de estudios socio-ecológicos a largo plazo donde existen las capacidades científicas y educativas para apreciar ecológica y éticamente a los pequeños co-habitantes con quienes compartimos el planeta.

\section{AGRADECIMIENTOS}

El autor principal agradece a la Beca de Magíster otorgada por el Instituto de Ecología y Biodiversidad (IEB), proyectos ICM, P05-002, CONICYT PFB-23, y al proyecto FONDECYT 11130451. La compleción de este trabajo ha sido apoyada también por los proyectos de Apoyo a Centros Científicos y Tecnológicos de Excelencia con Financiamiento Basal PIA- 


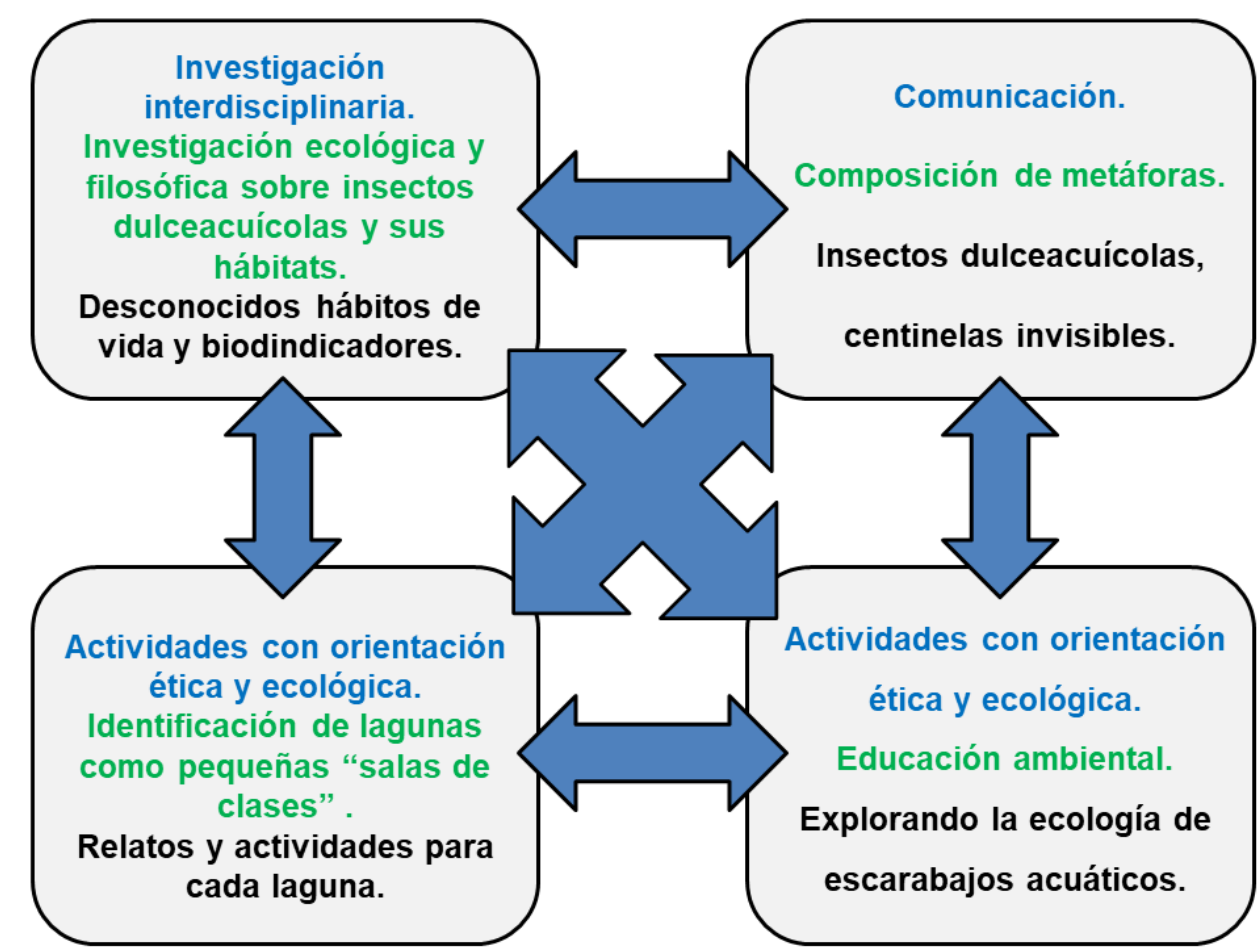

Fig. 5. Diagrama conceptual de los cuatro pasos de la Filosofía Ambiental de Campo (FILAC). En azul se describe el paso, el objetivo y/o método en verde, y el resultado en negro.

Las flechas indican la interconexión e interrelación de cada paso. Figura adaptada de Rozzi et al. (2010).

CONICYT AFB170008 al Instituto de Ecología y Biodiversidad (IEB-Chile) e IFI-CORFO a la Universidad de Magallanes (UMAG). Agradecemos las ediciones de Francisca Massardo y los comentarios de dos revisores anónimos. Este artículo es una contribución del Programa de Conservación Biocultural Subantártica (conjuntamente coordinado por UMAG e IEB en Chile y la Universidad de North Texas en EE.UU.) y, en particular, del Laboratorio Wankara de Ecosistemas Dulceacuícolas Antárticos y Subantárticos.

\section{BIBLIOGRAFÍA}

Bradbury, R. H., Vanderlann, J. D., \& Green, D. G. (1996). The idea of complexity in ecology. Senckenbergiana maritima, 27, 89-96.

Cardoso, P., Erwin, T. L., Borges, P. A., \& New, T. R. (2011). The seven impediments in invertebrate conservation and how to overcome them. Biological Conservation, 144(11), 2647-2655.

Castro, V. (2007). A propósito de Raíces históricas de nuestra crisis ecológica: Cuarenta años después, ¿qué hay de nuevo? Edición Especial Ética Ambiental. Revista Ambiente y Desarrollo, 23(1), 95-97.

Collen, B., Böhm, M., Kemp, R., \& Baillie, J. E. (2012). Spineless: Status and Trends of the World's Invertebrates. Zoological Society of London, United Kingdom: Zoological Society of London.

Contador, T. A. (2011). Benthic macroinvertebrate of temperate, sub-Antarctic streams: effects of altitudinal zoning and temperature on the phenology of aquatic insects associated to Róbalo river, Navarino Island $\left(55^{\circ} \mathrm{S}\right)$, Chile. PhD Dissertation, University of North Texas.

Contador, T. A., Kennedy, J. H., Ojeda, J., Feinsinger, P., \& Rozzi, R. (2014a). Ciclos de vida de insectos dulceacuícolas y cambio climático global en la ecorregión subantártica de Magallanes: investigaciones ecológicas a largo plazo en el Parque Etnobotánico Omora, Reserva de Biosfera Cabo de Hornos (55S). Revista Bosque, 35(3), 429-437.

Contador, T., Kennedy, J. H., \& Rozzi, R. (2012). The Conservation Status of South American Aquatic Insects in the Literature. Biodiversity and 
Conservation, 21, 2095-2107.

Contador, T., Kennedy, J. H., Rozzi, R., \& Villarroel, J. O. (2015). Sharp altitudinal gradients in Magellanic Sub-Antarctic streams: patterns along a fluvial system in the Cape Horn Biosphere Reserve ( $\left.55^{\circ} \mathrm{S}\right)$. Polar Biology, 38(11), 1853-1866.

Contador, T., Méndez, M., Medina, Y., Ojeda, J., Barroso, O., Crego, R., González, J., \& van de Maele, M. (2014b). Río Róbalo Comunidad de Vida. Contador \& Arriagada ediciones. $199 \mathrm{pp}$.

Contador, T., Rozzi, R., Kennedy, J., Massardo, F., Ojeda, J., Caballero, P.,... \& Lazzarino, S. (2018). Sumergidos con lupa en los ríos del Cabo de Hornos: Valoración ética de los ecosistemas dulceacuícolas y sus cohabitantes. Magallania, 46(1), 183-206.

Dallas, H. F., \& Rivers-Moore, N. A. (2012). Critical thermal maxima of aquatic macroinvertebrates: towards identifying bioindicators of thermal alteration. Hydrobiologia, 679(1), 61-76.

Ernst, C., Vinke, K., Giberson, D., \& Buddle, C. M. (2013). Insects in Education: Creating tolerance for some of the world's smallest citizens. In R. H. Lemelin (Ed.), The management of insects in recreation and tourism (pp. 289-305). Ontario: Cambridge University Press.

Fernández, H. R., \& Domínguez, E. (2001). Guía para la Determinación de los Artrópodos Bentónicos Sudamericanos. Tucumán, Argentina: Editorial Universitaria Tucumán.

Fischang, W. J. (1976). Another wasted resource. The American Biology Teacher, 38, 204-204.

Hallman, C., Sorg, M., Jongejans, E., Siepel, H., Hofland, N., Schwan, H., Stenmans, W., Müller, A., Sumser, H., Hörren, T., Goulson, D., \& de Kroon, H. (2017). More than 75 percent decline over 27 years in total flying insect biomass in protected areas. PLOS ONE, $12(10), e 0185809$

Hargrove, G. (2007). El debate de Lynn White Jr. Especial: Ética Ambiental. Revista Ambiente y Desarrollo, 23(1), 90-92.

Hedin, L. O., Armesto, J. J., \& Johnson, A. H. (1995). Patterns of nutrient loss from unpolluted, old-growth temperate forests: Evaluation of biogeochemical theory. Ecology, 76(2), 493-509.

Holzer, J. M., Adamescu, M. C., Bonet-García, F. J., DíazDelgado, R., Dick, J., Grove, J. M., Rozzi, R., \& Orenstein, D. E. (2018). Negotiating local vs. global needs in the International Long Term Ecological Research (ILTER). Network's Socio-Ecological
Research Agenda. Environmental Research Letters, 13, 105003. https://doi.org/10.1088/17489326/aadec8

Likens, G. E. (1991). Toxic winds, whose responsibility? En F. G. Bormann \& S. R. Kellert (Eds.), Ecology, Economics, and Ethics: the broken circle (pp. 136152). Connecticut: Yale University Press.

Lockwood, J.A. (2013). The Infested Mind: Why Humans Fear, Loathe and Love Insects. Oxford University Press. $240 \mathrm{pp}$.

Maass, M., Balvanera, P., Bourgeron, P., Equihua, M., Baudry, J., Dick, J.,... \& Vadineanu, A. (2016). Changes in biodiversity and trade-offs among ecosystem services, stakeholders, and components of well-being: the contribution of the International Long-Term Ecological Research network (ILTER) to Programme on Ecosystem Change and Society (PECS). Ecology and Society, 21(3). 31. http:// dx.doi.org/10.5751/ES-08587-210331

Mach, P. M., Winfield, J. L., Aguilar, R. A., Wright, K. C., \& Verbeck, G. F. (2016). A portable mass spectrometer study targeting anthropogenic contaminants in SubAntarctic Puerto Williams, Chile. International Journal of Mass Spectrometry, 422, 148-153.

May, R. H. Jr. (2018). Land grabbing and violence against environmentalists. En R. Rozzi, R. May, S. F. Chapin, F. Massardo, M. Gavin, I. Klaver, A. Pauchard, M. A. Núñez \& D. Simberloff (Eds.), From biocultural homogenization to biocultural conservation ( $\mathrm{pp}$. 109-123). Dordrecht: Springer.

McKinney, L. (1999). High rates of extinction and threat in poorly studied taxa. Conservation Biology, 13, 1273-1281.

Méndez, M., Rozzi, R., \& Cavieres, L. (2013). Flora vascular y no-vascular en la zona altoandina de la isla Navarino $\left(55^{\circ} \mathrm{S}\right)$, Reserva de Biosfera Cabo de Hornos, Chile. Gayana Botánica, 70(2), 337-343.

Merritt, R. W., \& Cummins, K. (2008). An introduction to the aquatic insects of North America. Dubuque, IA: Kendall/Hunt Publishing Company.

Mittermeier, R. A., Mittermeier, C. G., Brooks, T. M., Pilgrim, J. D., Konstant, W.R., Fonseca, G. A. B., \& Kormos, C. (2003). Wilderness and biodiversity conservation. Proceedings of the National Academy of Sciences, 100, 10309-10313.

New, T. R., \& Samways, M. J. (2014). Insect conservation in the southern temperate zones: an overview. Austral Entomology, 53, 26-31.

Proctor, J. D., \& Larson, B. M. (2005). Ecology, complexity, 
and metaphor. BioScience, 55(12), 1065-1068.

Ramírez, A., \& Gutiérrez-Fonseca, P. E. (2014). Estudios sobre macroinvertebrados acuáticos en América Latina: avances recientes y direcciones futuras. Revista de Biología Tropical, 62, 9-20.

Rendoll Cárcamo, J. (2018). Variación estacional y altitudinal en la diversidad, distribución y tolerancias térmicas de macroinvertebrados dulceacuícolas sub-Antárticos: en la búsqueda de bioindicadores térmicos para el cambio climático global. Tesis de Magíster, Universidad de Magallanes.

Rendoll, J., Contador, T., Gañán, M., Pérez, C., Maldonado, A., Convey, P.,... \& Rozzi, R. (2019). Altitudinal gradients in Magellanic sub-Antarctic lagoons: the effect of elevation on freshwater macroinvertebrate diversity and distribution. PeerJ, 7, e7128. http:// doi.org/10.7717/peerj. 7128

Rozzi, R. (2007). Seres vivos más que "recursos naturales". Edición Especial: Ética Ambiental. Revista Ambiente y Desarrollo, 23(1), 87-89.

Rozzi, R. (2012). Biocultural ethics: the vital links between the inhabitants, their habits and regional habitats. Environmental Ethics, 34, 27-50.

Rozzi, R. (2013). Biocultural Ethics: From biocultural homogenization toward biocultural conservation. En R. Rozzi, S. T. A. Pickett, J. J. Armesto, \& J. B. Callicott (Eds.), Linking ecology and ethics for a changing world: Values, philosophy, and action, Ecology and Ethics (pp. 9-32). New York: Springer.

Rozzi, R. (2015). Implications of the biocultural ethic for Earth stewardship. En R. Rozzi, F. S. Chapin, J.B. Callicott, S. T. A. Pickett, M. E. Power, J. J. Armesto, R. H. May Jr. (Eds.), Earth stewardship: Linking ecology and ethics in theory and practice (pp. 113-136). Dordrecht: Springer.

Rozzi, R., Anderson, C. B., Pizarro, J. C., Massardo, F., Medina, Y., Mansilla, A., Kennedy, J. H., Ojeda, J., Contador, T. A., Morales, V., Moses, K., Poole, A., Armesto, J. J., \& Kalin, M. T. (2010). Field environmental philosophy and biocultural conservation at the Omora Ethnobotanical Park: methodological approaches to broaden the ways of integrating the social component ("S') in LongTerm SocioEcological Research (LTSER) Sites.
Revista Chilena de Historia Natural, 83, 27-68.

Rozzi, R., Armesto, J., Goffinet, B., Buck, W., Massardo, F., Silander, J., Arroyo, M., Russel, S., Anderson, C. B., Cavieres, L. A., \& Callicott, J. B. (2008). Changing lenses to assess biodiversity: patterns of species richness in Sub-Antarctic plants and implications for global conservation. Frontiers in Ecology, 6, 131-137.

Rozzi, R., Armesto, J. J., Gutiérrez, J., Massardo, F., Likens, G., Anderson, C.,... \& Kalin, M. T. (2012). Integrating ecology and environmental ethics: Earth stewardship in the southern end of the Americas. BioScience, 62(3), 226-236.

Rozzi, R., Chapin, F. S., Callicott, J. B., Pickett, S. T. A., Power, M. E., Armesto, J. J., \& May R. H. Jr. (2015). Earth Stewardship: Linking Ecology and Ethics in Theory and Practice. Springer, Dordrecht: Netherlands.

Rozzi, R., Massardo, F., Berghoefer, A., Anderson, C. B., Mansilla, A., Mansilla, M., Plana, J., Berghoefer, U., Araya, P., \& Barros, E. (2006a). The Cape Horn Biosphere Reserve. Ediciones Universidad de Magallanes, Punta Arenas, Chile.

Rozzi, R., Massardo, F., Anderson, C., Heidinger, K., Silander J. Jr. (2006b). Ten Principles for biocultural conservation at the southern tip of the Americas: The approach of the Omora Ethnobotanical Park. Ecology and Society, 11(1), 43. [online] URL: http://www. ecologyandsociety.org/vol11/iss1/art43/

Samways, M. J. (2015). Future-proofing insect diversity. Current Opinion in Insect Science, 12, 71-78.

Sánchez-Bayo, F., \& Wyckhuys, K. A. (2019). Worldwide decline of the entomofauna: A review of its drivers. Biological conservation, 232, 8-27.

Schüttler, E., Crego, R. D., Saavedra-Aracena, L., SilvaRodríguez, E. A., Rozzi, R., Soto, N., \& Jiménez, J. E. (2019). New records of invasive mammals from the sub-Antarctic Cape Horn Archipelago. Polar Biology, 42(6), 1093-1105.

White Jr, L. (1967). Raíces históricas de nuestra crisis ecológica. Traducido al español por José Tomás Ibarra, Francisca Massardo y Ricardo Rozzi en Ética Ambiental, Edición Especial de Revista. Ambiente y Desarrollo, 23(1), 78-86. 